\title{
Immunological Response to Injury and Grafting in The Central Nervous System
}

\author{
K.L. Boyer, A. Ansari, W.C. Chan, C.R. Freed* and R.A.E. Bakay \\ Emory University School of Medicine, Department of Neurosurgery, Atlanta, Georgia, *University of \\ Colorado, Denver, Colorado, USA
}

\section{INTRODUCTION}

The rhesus monkey (macaca mulatta) is being used in the development of a non-human primate model that will study neural tissue transplantation as a potential treatment for degenerative diseases of the central nervous system (CNS). The overall quality of this model rests on several factors, including survival, growth, and long-term function of the grafted tissues.

Characterization of the immunological response of the CNS to allografts in primates is essential in the development of effective neural transplantation techniques. The apparent lack of systemic humoral and cellular sensitization as well as local tissue cytotoxic activity is critical in the ultimate survival of grafted tissues and efficacy of treatment. Appreciation of recipient humoral and cellular responses to transplanted tissues should maximize graft survival and limit graft rejection via immune system suppression. Host sensitization to donor specific tissues (systemic humoral and cellular response) and surgical site reaction (mononuclear cell infiltration) are of primary concern. Means by which to monitor host response to grafted tissues may then be determined and allow assessment of possible modalities of immunological manipulation.

\section{METHODS}

Immunological studies were performed on surgical site tissues (autografts and non-grafted), peripheral blood samples, and cerebrospinal fluid (CSF) from the rhesus monkeys. Both fetal mesencephalic allografts and adrenal medullary autografts were evaluated. Peripheral blood was obtained from fetal grafted Bonnett monkeys (macaca radiata) and the donor parent from the University of Colorado.

Ten monkeys at the Yerkes Regional Primate Research Center were evaluated for CNS grafting. These monkeys were trained to perform specific tasks and were injected with 1-methyl-4phenyl-1,2,3,6-tetrahydropyridine (MPTP, 0.4 $\mathrm{mg} / \mathrm{kg}$ ) via left internal carotid to induce parkinsonism. After three to four months of functional impairment quantitation, fetal mesencephalic allografts $(\mathrm{N}=2)$ or adrenal medullary autografts $(\mathrm{N}=4)$ were transplanted to the left caudate nucleus. Sham operations were performed on four animals. Prior to operation, CSF was sampled via percutaneous cisterna magna punctures and peripheral blood was drawn monthly. Functional impairment was again quantified for eight to twelve months during which time CSF was again sampled monthly and peripheral blood was assayed at one, three, and six months. The monkeys were sacrificed at eight to twelve months post-operatively for histological and immunocytochemical study of graft site.

Leukocyte and erythrocyte counts were performed on all CSF samples. Surface markers specific for mononuclear cells were used to identify cellular infiltrates in tissues one week, one month, and one year after surgery. Markers included antibodies to $\mathrm{CD} 2, \mathrm{CD} 3, \mathrm{CD} 4, \mathrm{CD} 8$, CD12, CD20, CD25, and CD68. Relative evaluation of major histocompatibility complex (MHC) class I and class II antigenic expression was also performed as were functional studies to detect donor specific cytotoxic T-cells, and levels of NK and LAK cells. Serum samples were also assayed for presence of donor specific cytotoxic antibodies. 


\section{RESULTS}

Peripheral blood assays from both fetal mesencephalic and adrenal medullary grafted animals revealed no significant changes in the frequency of mononuclear cell subsets, including total T-cells, CD4 (helper T-cells), CD8 (suppressor T-cells), B-cells, or macrophages when preoperative samples were compared to post-transplant samples. Cytotoxicity evaluations failed to disclose any sign of humoral or cellular donor specific sensitization in hosts receiving mesencephalic allografts. Functional evaluations failed to demonstrate changes in the relative levels of NK or LAK cells in experimental as compared with non-transplanted surgical control samples.

CSF samples throughout the study showed no significant mononuclear cell count elevation.

Surface immunoreactivity utilizing antibodies to mononuclear cell specific antigens disclosed evidence of helper T-cell, suppressor T-cell, activator T-cell, B-cell, and macrophage activity in tissues from ungrafted operative site at one week, one month, and up to one year postoperatively. There appeared to be an overall decrease in surface marker evidence with time. Interestingly, the same degree of response appeared to occur whether or not an autograft was implanted to the surgical site. Allograft tissue data is not yet available.

Relative to splenic tissues, MHC class I antigens were expressed at very low levels and MHC class II antigen levels were undetectable in brain tissues taken from surgical sites with or without graft at one week, one month, and one year postoperatively.

\section{CONCLUSION}

These results suggest that allografts to the CNS do not evoke donor specific lymphocytotoxic sensitization. There is evidence for mononuclear cellular reaction in the surgical site whether or not a graft is placed. MHC class I antigens have been detected at very low levels in the brain tissue. Future studies include evaluation of host sensitization to subsequent antigenic challenges from the donor and suppression of immunological response using various pharmacological means.

Supported by Veterans Administration merit award, USPHS Grant RR00165 and NINCDS N24340. 

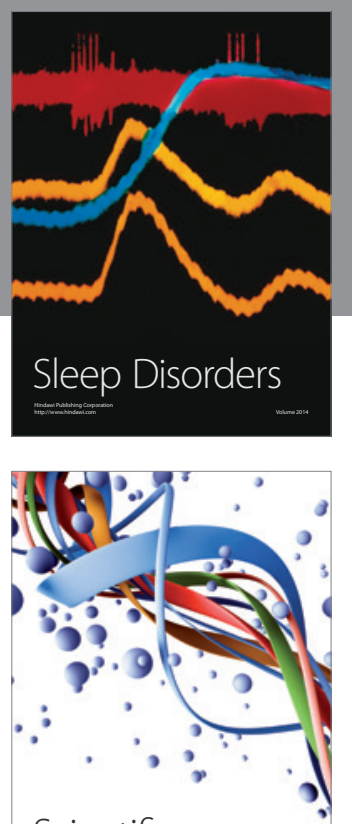

Scientifica
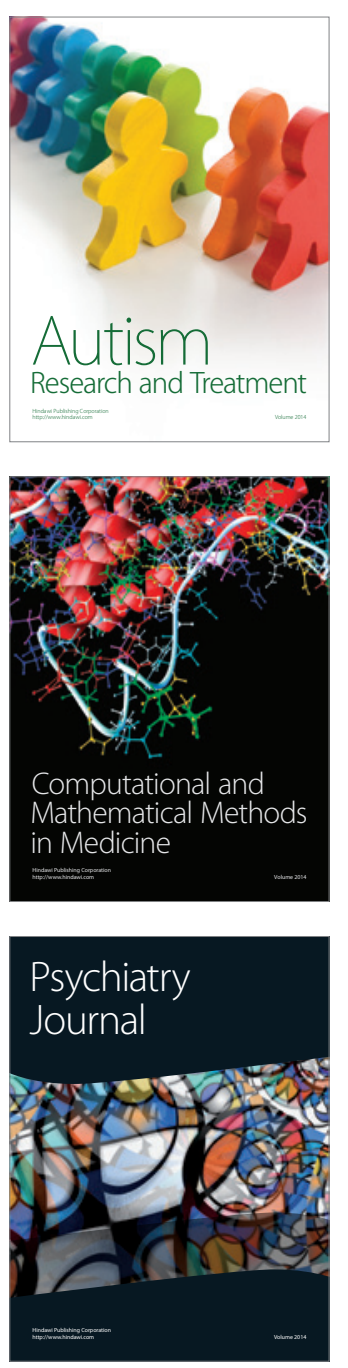
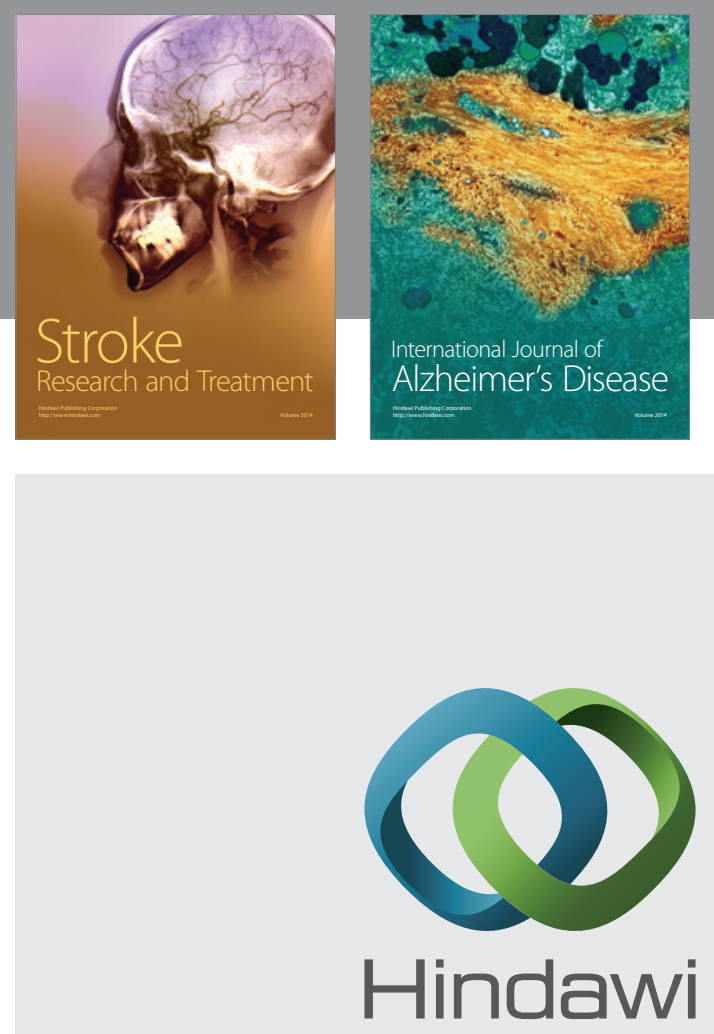

Submit your manuscripts at

http://www.hindawi.com
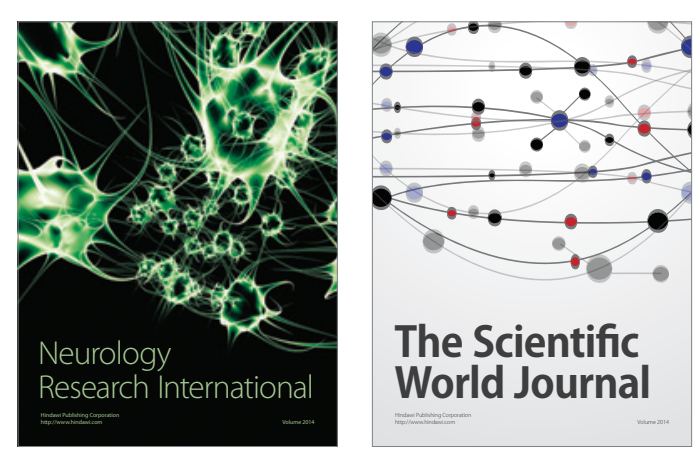

The Scientific World Journal

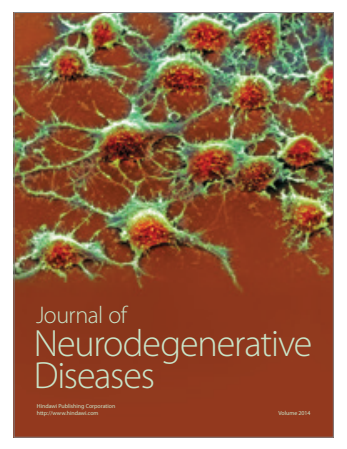

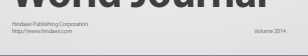

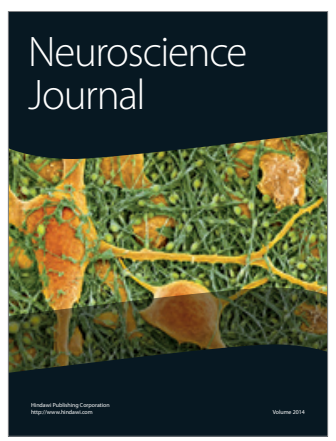

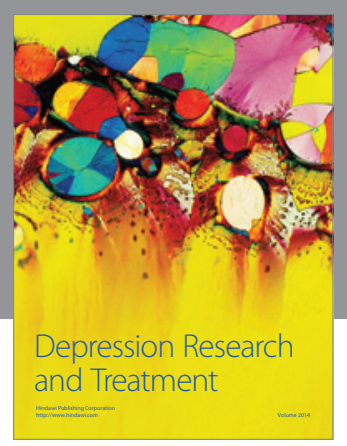
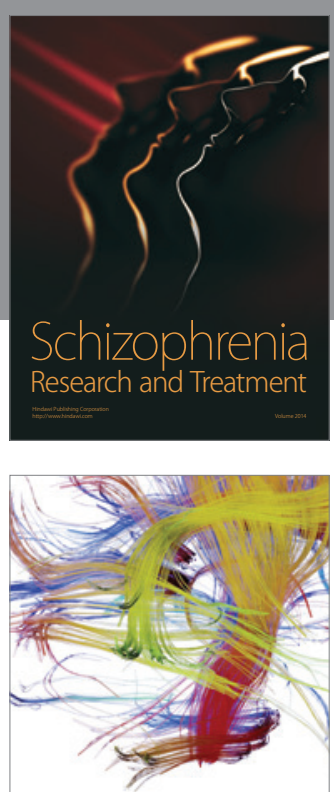

Brain Science

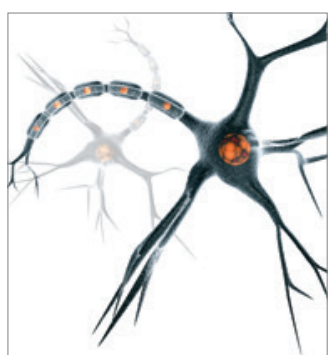

Neural Plasticity
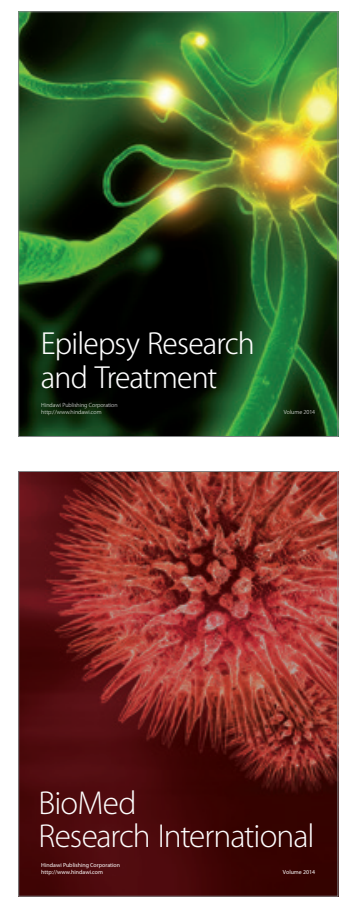

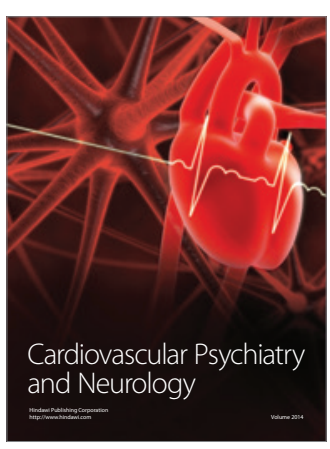

Parkinson's

Disease
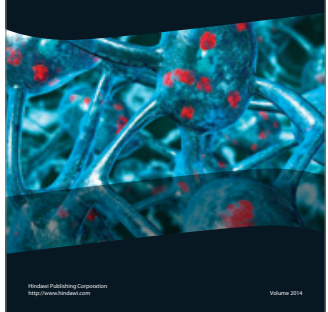